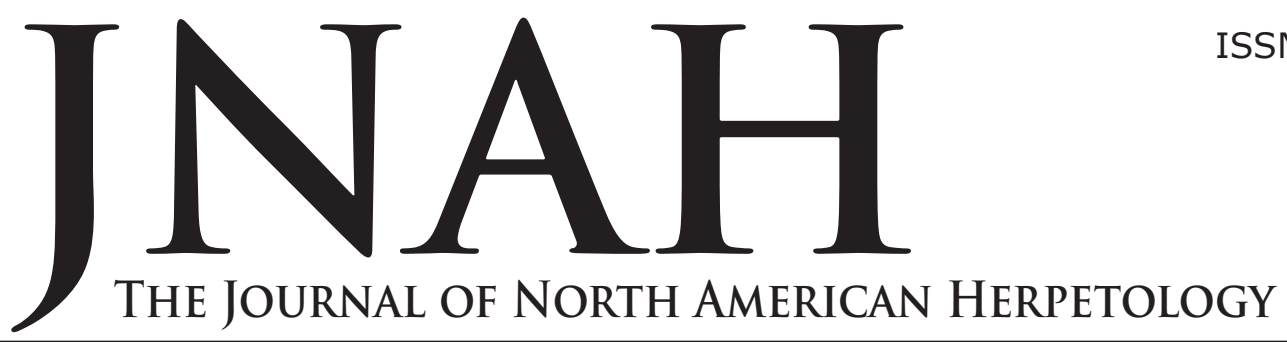

ISSN 2333-0694

Volume 2020, Number 1

2 July 2020

journals.ku.edu/jnah

\title{
THE FOURTH INTERNATIONAL SYMPOSIUM ON RANAVIRUSES: SUMMARY OF NORTH AMERICAN HERPETOLOGICAL CONTENT AND POINTS OF INTEREST
}

\author{
LAUREN V. ASH ${ }^{1}$, RACHEL E. MARSCHANG ${ }^{2}$, \\ JOLIANNE M. RIJKS ${ }^{3}$, AMANDA L. J. DUFFUS ${ }^{4,5}$ \\ ${ }^{1}$ Department of Biology, University of Vermont, Burlington, VT, USA \\ 2 Laboklin GmbH and Co. KG, Bad Kissingen, Germany \\ ${ }^{3}$ Dutch Wildlife Health Centre, Department of Pathobiology, Faculty of Veterinary Medicine, Utrecht \\ University, Utrecht, Netherlands \\ ${ }^{4}$ Department of Biology and Physical Sciences, Gordon State College, Barnesville, GA, USA \\ ${ }^{5}$ Corresponding Author: Tel: (678) 359-5464; Fax: (678) 359-5850; Email: aduffus@gordonstate.edu
}

Ranaviruses are large double-stranded DNA viruses from the family Iridoviridae. They are globally distributed and are currently known to affect fish, reptiles and amphibians. In North America, ranaviruses are also widely distributed, and cause frequent morbidity and mortality events in both wild and cultured populations.

In May 2017, the Fourth International Symposium on Ranaviruses was held in conjunction with the Tenth International Symposium on Viruses of Lower Vertebrates, in Budapest, Hungary at the historical Veterinary University (https://www.ranavirus.org/wp-content/ uploads/2017/02/ISR2017 Budapest AbstractBook. pdf). There were over 56 participants from 12 countries with diverse areas of expertise, including wildlife professionals, researchers, veterinarians, and policy makers. Events over the four days of the conference included topical lectures, oral presentations, poster presentations, topical discussions, a workshop, the Global Ranavirus Consortium's general meeting, a dinner river cruise, and field trips.

Despite the fact that the Symposium was overseas, there was a significant amount of content that focused on or directly impacted North American herpetofaunal species. In total, there were eight oral presentations and one poster that focused specifically on North American species. We also highlight two general presentations which were of direct consequence to all herpetofaunal species.

E. Davis Carter, University of Tennessee, presented his findings on the estimated prevalence of ranaviruses in amphibian communities in Great Smokey Mountains National Park. Carter and colleagues conducted a surveillance study of the amphibian communities in Gourley Pond, which has had recorded ranavirus outbreaks since 1999, and Little Gourley Pond, a small pond nearby. Between February and May 2016, they captured 1,972 adults of 16 amphibian species and two larval species. They detected a ranavirus at a low prevalence in adults of Wood Frogs (Lithobates sylvaticus), Eastern Spotted Newts (Notophthalmus viridescens), and Spotted Salamanders (Ambystoma maculatum). However, the hydroperiod of the ponds was short, and both were dry by April before any outbreaks occurred. The next year, between February and March 2017, they captured 102 adults of 11 amphibian species. No larval species were captured because the ponds filled up late in the season. They concluded that variable hydroperiods might be contributing to population declines in the ponds, leading to egg mass deaths and limited recruitment. Additionally, they speculated that disease persistence in adults, with a sufficient hydroperiod, could lead to virus amplification in susceptible larvae, and, subsequently, to further population declines.

Roberto Brenes, Carroll University, and Joe-Felix Bienentreu (presented by David Lesbarrères from Laurentian University), separately investigated how amphibian community composition alters ranavirus outbreaks. Bienentreu and colleagues observed disease prevalence at ponds with low diversity in Canada. Community composition across ponds varied in relative abundance of the Wood Frog, the Boreal Chorus Frog (Pseudacris maculata) and the Canadian Toad (Anaxyrus hemiophrys). They found adult and metamorph Wood Frogs and Boreal Chorus Frogs, but not Canadian Toads, were infected with a ranavirus. They investigated the genetic diversity of ranavirus strains across ponds and detected two strains of an FV3-like virus. Strain A was observed in amphibian communities with all three species; interestingly, strain B was observed in ponds with only Wood Frogs. The study conducted by Brenes and colleagues used mesocosms to investigate the unequal transmission of ranaviruses 
among hosts. They created two amphibian communities, one with coastal plain species (Gopher Frog, Lithobates capito, Upland Chorus Frog, Pseudacris feriarum, and Southern Toad, Anaxyrus terrestris larvae) and one with Appalachian species (Wood Frog, Upland Chorus Frog, and Spotted Salamander larvae). Using treatments of either one, all, or no species initially exposed to FV3, they discovered that Wood Frogs not only caused an outbreak of ranaviral disease in Upland Chorus Frog larvae, but also amplified the mortality of Spotted Salamander larvae to twice the mortality when directly exposed to the virus. Additionally, each of the coastal plains species was able to cause ranavirus outbreaks with $40 \%$ mortality or more in the previously unexposed cohabitant species. These studies highlight the influence of host community composition on virus strains present and on outbreak dynamics and impact.

Claytor and colleagues, presented by Thomas Waltzek from the University of Florida, investigated the genome sequences of ranaviruses from different outbreaks in the same ranaculture facility, which resulted in American Bullfrog (Lithobates catesbeianus) die-offs in both 1998 and 2006. Claytor et al. found that the virus (named RCV-Z) responsible for the 1998 outbreak was closely related to the Common Midwife Toad Virus (CMTV), a ranavirus previously thought to be restricted to Europe and China. Additionally, they showed that the 2006 virus (named $\mathrm{RCV}-\mathrm{Z2}$ ) resulted from a chimeric ranavirus strain, with CMTV-like genes in a predominately FV3 genomic background. They indicated this is most likely due to recombination between the CMTV-like and wildtype FV3 ranaviruses. This is the first confirmed report of a chimeric ranavirus. Both RCV-Z and RCV-Z2 showed higher virulence than wild-type FV3, a possible indication that CMTV contains genes directly related to an increase in pathogenicity. Ranaculture facilities, where specimens are kept in high densities and there is great turnover, may produce more virulent recombinant ranavirus strains that may then spread and pose a threat to amphibian communities in the wild.

Rebecca Hardman, University of Tennessee, presented her findings on the prevalence of ranaviruses and $B a-$ trachochytrium spp. in the Hellbender (Cryptobranchus alleganiensis) populations found in Arkansas and Tennessee. Hardman and colleagues note that there are currently two distinct subspecies of hellbender, C. a. bishopi, found in Arkansas, and C. a. alleganeinsis, found in Tennessee. They collected tissue samples and swabs from individuals in both Tennessee and Arkansas. Infection with ranaviruses was lower in prevalence for both species than infection with Batrachochytrium dendrobatidis. In Arkansas, C. a. bishopi had a ranavirus infection prevalence of $8.3 \%$, while in Tennessee C. a. alleganeinsis had a $14.7 \%$ prevalence. The presence of the two pathogens in these populations of our endangered North American giant salamanders is of conservation concern. Hardman et al. are currently examining the role of skin microbiomes in changes associated with clinical signs of disease in these animals.

Debra Miller, University of Tennessee, also studied hellbenders and ranaviruses. However, here Miller and colleagues examined the relationship between juvenile hellbenders exposed to a ranavirus alone or with the addition of another stressor, in this case $B$. dendrobatidis or glyphosate herbicide. Standard staining methods were used to examine the tissues from exposed animals for ranaviral disease, and immunohistochemical techniques to examine tissues for the presence of ranavirus virions. Miller and colleagues found that disease and virions were widespread in the tissue of the juvenile hellbenders that had been exposed to ranavirus at $22^{\circ} \mathrm{C}$. While they note that proportionally more animals died in the treatments that included a stressor, there were no differences noted in staining distribution or intensity with immunohistochemical methods. This is important as it provides insights into disease progression and the role of other stressors in the development of disease caused by ranavirus infection. Increasing our understanding of how multiple stressors interact with ranavirus infection will be important in developing mitigation and management plans for sensitive or endangered species, such as the hellbender.

Emily Hall, Vanderbilt University Medical Center (formerly of Washington State University), presented some intriguing work on ranavirus seasonal dynamics in Wood Frogs. Using environmental DNA (eDNA) sampling methods and larval sampling, Hall and colleagues examined several vernal wetlands in the Northeastern USA for the prevalence of ranaviruses over the course of their development. Interestingly, they found that the timing of the first detection of ranavirus in the populations had little effect on when a mortality event occurred. In addition to this, it was found that ranavirus prevalence in larvae could reach $\geq 75 \%$ and the mortality event could occur up to six weeks after this maximum had been reached. Mortality events were associated with water temperature and larval developmental stage, rather than time of introduction and ranavirus prevalence in the population. Understanding the seasonal dynamics of ranavirus infections, morbidity, and mortality events in wild populations is extremely important if we are going to attempt to mitigate the effects of these outbreaks and manage populations effectively.

Angela Peace, Texas Tech University, and colleagues also used Wood Frogs as their model system. Matthew Gray, University of Tennessee, presented on how Peace et al. used a mathematical approach to understand how density dependent and frequency dependent transmission affect the transmission pathways of a ranavirus in this system. They developed a traditional susceptible-infection (SI) type compartmental model for ranavirus transmission that includes the latent phase, and accounts for different modes of transmission (direct contact, environmental via virions shed into the water, necrophagy). The model assumes that the likelihood of mortality caused by ranaviral disease increases with time, as well as the likelihood of infection transmission as ranaviral disease progressed. The model was parameterized using empirical estimates of laboratory collected data. Regardless of density dependent or frequency dependent transmission, the most important transmission pathway was environmental transmission of virions in water across a range of typical Wood Frog tadpole densities. The model also estimated that the invasion potential $\left(R_{0}\right)$ of the ranavirus they used was greater than any other previously reported amphibian pathogen. Incidentally, the virus they used in their experiments was the chimeric FV3CMTV virus discussed by Claytor and Waltzek. The model presented by Peace and colleagues is an important step in understanding the transmission dynamics of ranaviruses in a single species anuran system, and illustrates the potential population impacts of chimeric ranaviruses.

There was a single, but notable, poster presentation detailing ranavirus infection in North American herpeto- 
fauna. Lauren Ash, University of Vermont, presented the findings of the first survey for ranaviruses in Vermont. Ash and colleagues repeatedly sampled 18 ponds from May to August 2016 on a biweekly basis. They amassed a total sample size of over 1800 individuals, from which a subset of 220 were screened for the presence of ranaviruses using quantitative PCR. While they did not witness any mass mortality at the sites, they did find that a ranavirus was present in four species (Eastern Spotted newts; Green Frogs (Lithobates clamitans); Northern Leopard Frogs (Lithobates pipiens); Wood Frogs) in four of the ponds that were studied. Additionally, they found that ranavirus prevalence was greatest in July and August. They hope that their work will be used to inform conservation efforts and reduce the human-enhanced spread of the virus to other naïve populations in the state.

Jesse Brunner, Washington State University, presented on diagnostic methods to screen for ranavirus infection in herpetological fauna. Since monitoring for ranaviruses in trade and captive populations requires non-lethal sampling, Brunner and colleagues evaluated the diagnostic performance of tail or toe clips, swabs, and eDNA when compared to liver tissue samples using quantitative PCR. They experimentally infected 390 American Bullfrog tadpoles with 1 of 3 doses and sampled at 10 time points. Overall, they found that performance changed with time after infection, becoming less sensitive and more specific (with fewer false positives) over time. Detection rates for all non-lethal samples with low-level infection were generally poor. An eDNA-approach had higher specificity than the use of swabs and clips, and while eDNA was less sensitive, it was more efficient in detecting low-level, incidental, infections. Brunner and colleagues emphasized that an eDNA-based approach to ranavirus, and potentially other pathogen surveillance, can be efficiently implemented, especially in captive settings.

Matthew Gray, University of Tennessee, presented on handling methods that impact ranavirus transmission. Gray and colleagues conducted an experiment to determine whether poor biosecurity practices, such as lack of glove changes and co-housing, could facilitate ranavirus transmission and increase mortality in Wood Frog tadpoles. To test this, they co-housed tadpoles for 15, 30, and 60 minutes at 10,20, and 40\% ranavirus prevalence, and in another experiment, did not change gloves between handling consecutive tadpoles, with 10, 20, and $40 \%$ of tadpoles initially infected. When co-housed for 10 minutes at $10 \%$ prevalence, uninfected tadpoles experienced $50 \%$ mortality, and no glove change with $10 \%$ of tadpoles initially infected resulted in $73 \%$ mortality. These results highlight why it is crucial for biologists to house animals separately and to use and change gloves, regardless of the nature of research being conducted.

During the symposium, Jesse Brunner held a workshop on how to use the Global Ranavirus Reporting System (GRRS). The GRRS is an online epidemiological database (https://mantle.io/grrs) that maps the known distribution of ranaviruses around the globe. The system is very flexible, and has a multitude of possible uses. The GRRS is currently being populated with published records of ranavirus presence/absence data thanks to a Wildlife Disease Association Small Grant. It is hoped that the GRRS will become an invaluable tool to managers, researchers, conservation professionals, and veterinarians.

At the end of the Symposium, participants attended one of two offered field trips. Attendees of the first field trip traveled to the Meadow Viper Conservation Centre in
Kiskunság National Park. There, the center has bred and released over 700 endangered Meadow Vipers (Vipera ursinii) since its establishment. Participants also attended a Pusta horse show before visiting the Upper-Kiskunság Plain. Attendees of the second field trip traveled to Sashegy (Eagle Hill) Protected Area in the Danube-Ipoly National Park, where they walked along dolomite slopes to observe a small Fire Salamander (Salamandra salamandra) population in the outskirts of the forest.

The Fourth International Symposium was supported with generous funding from the Global Ranavirus Consortium (GRC), Facultas Scientificarum Veterinarium Budapestiensis (the Veterinary University, Budapest), the US Department of Agriculture, the National Science Foundation (USA), the Association of Reptilian and Amphibian Veterinarians (ARAV), BioCenter (Hungary), and Laboklin GmbH \& Co. KG (Germany).

The Fifth International Symposium on Ranaviruses (ISR) was held in June 2019 in Australia! Information about ISR 2019 is posted on the GRC's website (www. ranavirus.org) and was distributed through the GRC's listserv, which can be joined at http://listserv.utk.edu/ cgi-bin/wa?A0=GRC.

\section{ACKNOWLEDGEMENTS}

Thank you to Roberto Brenes, Jesse Brunner, E. Davis Carter, Matthew Gray, Rebecca Hardman, Emily Hall, David Lesbarrères, Debra Miller, and Thomas Waltzek for letting us summarize and present their work in this manuscript and for their helpful comments on earlier versions of this document. Finally, thank you to the local organizing committee, Prof. Dr. Mária Benkö, Prof. Dr. Balázs Harrach, and Dr. Tibor Papp, and to all the participants of the symposium.

\section{ADDENDUM}

Since the $4^{\text {th }}$ International Symposium on Ranaviruses, three of the presentations have been published, their references are:

Claytor, S.C., Subramaniam, K., Landrau-Giovanetti, N., Chinchar, V.G., Gray, M.J., Miller, D.L., Mavian, C., Salemi, M., Wisely, S., and T.B. Waltzek. 2017. Ranavirus phylogenomics: Signatures of recombination and inversions among bullfrog ranaculture isolates. Virology. 511:330-343.

Gray, M.J., Spatz, J.A., Carter, E.D., Yarber, C.M., Wilkes, R.P., and D.L. Miller. 2018. Poor biosecurity could lead to disease outbreaks in animal populations. PLoS One. 13:e0193234

Peace A., O'Regan S.M., Spatz J.A., Reilly P.N., Hill R.D., Carter E.D., Wilkes R.P., Waltzek T.B., Miller D.L., and M.J. Gray. 2019. A highly invasive chimeric ranavirus can decimate tadpole populations rapidly through multiple transmission pathways. Ecological Modelling. 410:108777 